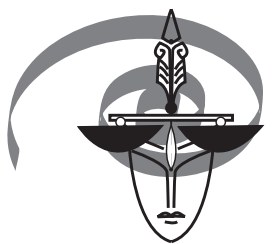

\title{
EUROPEAN
}

Volume $8 \cdot 2014 \cdot$ Number 4 (30)

DOI: 10.2478/ep-2014-0012

Jerzy Wojciechowski*

Faculty of Psychology

University of Warsaw

Warsaw

Poland

\section{Detection of concealed information with of the P300 potential amplitude analysis}

Key words: EEG and detection of deception, p-300 and deception

\section{Introduction}

Studies that make use of the bioelectric activity of the brain and falling back on the EEG are presented as an alternative to classical polygraph examinations, especially those that make use of the Guilty Knowledge Tests/Concealed Information Tests (GKT/CIT). Their main purpose is detection of concealed information through the analysis of the bioelectric activity of the brain in response to presented stimuli. The article aims at elucidating the main questions concerning the detection of concealed information with the use of P300 potential amplitude analysis, discussion of the chief advantages and disadvantages of the method, and presentation of a review of seminal studies.

\footnotetext{
"jerzy.wojciechowski@psych.uw.edu.pl.
} 


\section{A recording of the brain's bioelectrical activity: evoked potentials}

The first studies that made use of the EEG as an alternative for the polygraph focused on analysing the alpha rhythm, and especially its changes while lying (Obermann 1939). Beginning with the 1980s, special attention in studies of detecting concealed information has been devoted to the analysis of evoked potentials (EP): a distinctive, time-specific bioelectric response of the brain, also known as the event (or stimulus) related potential (ERP) (Schmitt 1993; Fabiani, Gratton, Coles 2000; Sosnowski 2000). As the spontaneous electric activity of the brain greatly exceeds the value of the evoked potential, ${ }^{1}$ there have always been certain difficulties related to its recording. The first to record successfully the evoked potential from the surface of the head was Dawson (1947). Replicating repeatedly the presentation of a stimulus in the form of an electric shock, he recorded each reaction visible on an oscilloscope with a still camera. The brightest spots on the photograph were connected to the places where the successive passages were ideally coherent. Known as superimposition, the method was the first to allow studying evoked potentials. Today, thanks to the use of amplifiers connected to computer data acquisition systems, averaging of the potentials, that is averaging a record after multiple presentations of the same phenomenon, is a common method (Jaśkowski 2008). Its foundation is the assumption that activity related to a stimulus will be correlated with it in time (in the successive presentations, it will have a similar latency: it will be present after the passage of the same period of time from the moment when the stimulus is presented) (ibidem). In turn, the spontaneous activity of the brain is treated as a particular noise, which, as random, should be eliminated during the averaging. In result, the changes related to the stimulus should be the only visible elements of the recording (Fabiani et al. 2000).

One of the most important divisions of the evoked potentials is the distinction between the exogenous and endogenous potentials. An exogenous (aka sensory) potential is one whose characteristics are related to the properties of the stimulus itself (its modality, frequency, and amplitude) (Fabiani et al. 2000; Sosnowski 2000). In turn, the term 'endogenous potential' is used to denote such a bioelectric reaction whose characteristics are related to the subject's own operation, and interaction between the stimulus and the subject (ibidem). It can be related among others to an emotional or cognitive reaction to an event or an unexpected change in the features of an event (Szelenberger 2001).

\footnotetext{
${ }^{1}$ It is a certain simplification to state that the evoked potential lies within the range of a few microvolts, while the spontaneous activity registered during a test reaches approximately $15 \mu \mathrm{V}$ (Fabiani et al. 2000).
} 
While Describing potentials, special attention is paid to positive and negative peaks in the course (Fabiani et al. 2000; Szelenberger 2001). The individual peaks are described for their polarity and delay after the presentation of the stimulus. This passage of time is known in literature as the latency of the peak (Fabiani et al. 2000; Schmitt 1993; Sosnowski 2000). Different researchers calculate the value of the amplitude of the evoked potential differently in their studies. The two most frequently applied methods are the baseline-to-peak method, and the peak-to-peak method (Fabiani et al. 2000). In the first, the amplitude of the potential is calculated from the baseline (zero level) set during the averaging to the maximum value of a given peak. In turn, the other one treats the value of the amplitude as the difference between the highest value of the given peak and the maximum value of the peak following it and bearing the opposite sign (Fabiani et al. 2000). The naming convention of individual peaks refers most often to their polarity and time of latency. For example, the P300 peak describes the positive peak (P) appearing approximately $300 \mathrm{~ms}$ after the presentation of the stimulus (ibidem). It is also worth mentioning that individual peaks are also known in literature as potentials, hence the P300 peak is also referred to as the P300 potential, with the names being used interchangeably. As this peak has the greatest significance from the point of view of studies of the detection of concealed information, the following subchapter is devoted to its characteristic traits, and especially to its cognitive correlates.

\section{P300 potential: cognitive correlates}

For the first time the P300 potential was described in the 1960s by Sutton and his team (1965). As was mentioned earlier, the name of the potential is related to its positive polarity and latency time of around $300 \mathrm{~ms}$. As, however, the studies proved that it can emerge even $800 \mathrm{~ms}$ after the emergence of the stimulus, some propose to call it the P-3 peak, i.e. the third positive peak from the emergence of the stimulus (Sosnowski 2000; Schmitt 1993).

Plenty of procedures were developed to investigate the P300 potential; the most important of these being (after Polich, Criado 2006): single stimulus task, oddball task, and three stimulus task procedures. In the single stimulus procedure, the task of the subject is to react to the only rare stimulus (target) that appears in different time spaces. The P300 potential is expected to follow its presentation. In the oddball procedure, a set of two different stimuli is presented in a random order. One of them is very frequent (a standard stimulus) and the other - very rare (target). It is expected that the P300 potential will emerge in response to the rare stimulus, unlike in the case of the frequent stimulus. 
The three-element procedure is akin to the oddball task, yet, besides the rare stimulus of the target type, and the frequent standard stimulus, there emerges the third rare stimulus: the distractor. The task of the subject is to react only to the target-type stimuli, ignoring all the others. This procedure should result in the emergence of two P300 potentials with different characteristics: a P3a subcomponent in response to the distractor, and a P3b subcomponent in response to the target-type stimulus. ${ }^{2}$

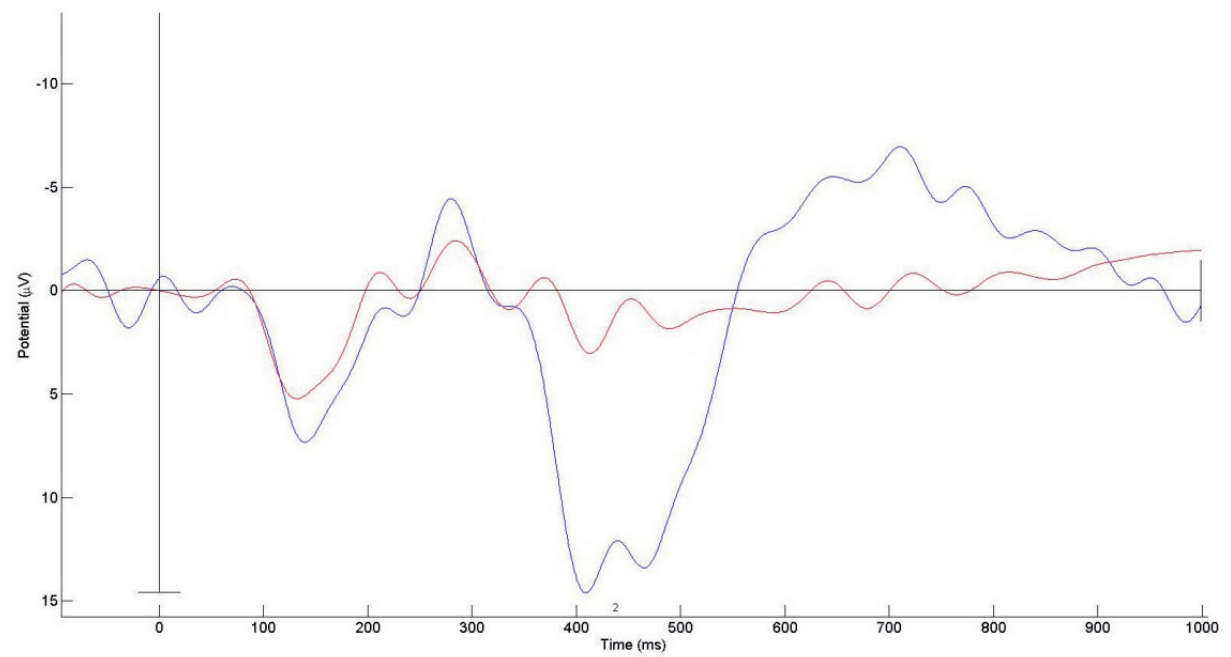

Figure 1. The P300 Potential (in blue) in response to a rare and significant stimulus (source: own study). According to a convention of evoked potential studies, positive values are situated below the OX axis.

Most investigations were devoted to the amplitude and latency of the P300 peak. Taking into account the localisation of electrodes, it was discovered that its amplitude is highest in the central line, with the value growing from the $\mathrm{Fz}$ lead over frontal lobes to the Pz lead over occipital lobes (Johnson 1993). Recapitulating the current state of the art on the P300 potential, Polich (2007) observed that the value of the P300 potential amplitude is related to a number of cognitive factors. Referring to the context-updating theory, he stressed that the emergence of the P300 potential in response to a new stimulus is related to the updating of the mental representation (Donchin 1981; for a summary of the theory, see: Polich 2007). It was observed (ibidem) that this concept of emergence of the $\mathrm{P} 300$ potential is close to the description of the rudiments

\footnotetext{
2 The subcomponents $\mathrm{P} 3 \mathrm{a}$ and P3b are described in detail further in the chapter.
} 
of the orientative response (the changes registered at the level of functioning of the autonomous nervous system, and in the behaviour of a person in response to the novelty or change of the stimulus) (see: Donchin 1981), with some researchers even making a direct reference to the concept of orientative response in interpreting a greater amplitude of the P300 potential, for example, in reference to the detection of concealed information (see: Vrij 2008). However, Donchin (1981) believes that the orientative response is a tactical one related to the planning of behaviour. For that reason, he claims that, unlike the reaction of the autonomous nervous system, it is hard to consider the P300 peak, a component of the orientative response. The researcher himself connected the P300 with the processing of significant information and memory processes: learning (ibidem). Later works (among others Karis, Fabiani, Donchin 1984) stated that the P300 potential is also the gauge of the process of recognition of a stimulus learnt earlier. It is also worth adding that the value of the P300 amplitude is related to the amount of attention resources available and involved in the execution of the task: the more demanding the task, the greater the amplitude of the potential. Yet in the case of simultaneous performance of two tasks - one which is to result in the emergence of the P300 potential (e.g. oddball procedure), and the other that engages attention resources - the increase of difficulty of the second task results in a drop in the amplitude of the P300 potential in oddball procedure (see: Polich 2007). Additionally, the value of the P300 potential amplitude depends on the distance between the successive stimuli and is inversely correlated to the frequency of presentation of the stimulus, and directly correlated with its significance: the more rare and important the presented stimulus is, the greater the amplitude of the P300 peak (Duncan-Johnson, Donchin 1977; Sosnowski 2000; Schmitt 1993). In turn, it is assumed that the time of latency is linked to the complexity of the stimulus: time of its processing and individual differences in the cognitive capacity of the individual (Polich 2007).

The basic information presented above refers to the P300 potential treated as a uniform phenomenon, independent of the procedures applied and cognitive mechanisms lying at its base. An increasing amount of attention is, however, paid to a more precise understanding of its both neuro-anatomical and cognitive grounds. A proposal was made to distinguish two subcomponents of the P300 potential, namely the P3a and P3b (Squires, Squires, Hillyard 1975) to distinguish the relatively quick (appearing already even between $220 \mathrm{~ms}$ and $280 \mathrm{~ms}$ ) positive potential emerging in a simple aural task in response to a rare stimulus with the highest amplitude in fronto-central leads (P3a) from a later positive potential (appearing after $320 \mathrm{~ms}$ ) present in tasks that require 
the processing of the main stimulus, and attaining its maximum in centraloccipital leads (P3b) (for a more detailed discussion, see also: Polich 2007). In his cognitive model of the grounds for the P300 potential, Polich (2003, after: Polich 2007; Polich and Criado 2006) combines the P3a subcomponent with the activity of the frontal lobes - the processes of attention employed in reaction to a rare of physically significant stimulus, and P3b - with the additional resources of attention and operations of memory updating related to the associative cortex. ${ }^{3}$

This allows an reflection that questions related to the study of the P300 potential are highly complex. The above results also from the fact that the P300 potential emerges in response to each task that requires the differentiation of the presented stimuli (Polich 2007). One of the attempts at the theoretical approach to the question of the P300 potential is the inhibition hypothesis, according to which it results from mechanisms involved in the inhibition of excessive brain activity (see: ibidem). Polich (ibidem) points out that there are a number of arguments in support of this hypothesis:

1) the need to inhibit unnecessary activity in response to stimuli with low probability of occurrence (which allows believing that they are also biologically significant) results in the emergence of a greater P300 amplitude

2) the performance of a difficult, competitive creatively engaging task limits the resources of attention and inhibition capacity, which results in a smaller P300 potential

3) the level of inhibition is modulated by excitement, which changes the accessibility of attention resources, which in turn influences the character of the P300 potential

4) latency of the P300 potential is linked to cognitive capacity (intelligence): it is related to the speed at which the redundant reaction is inhibited

5) with age, and in the case of dementias, the amplitude diminishes, and the latency of the P300 potential extends, which is linked to the weakening of the cortical processes that stimulate inhibition

6) the assumed system of P3a and P3b neurotransmitters impacts inhibition signals, which influences the $\mathrm{P} 300$ potential.

Due to the cognitive correlates of P300 potential amplitudes, this approach lies in the focus of interest of researchers interested in studying memory and concealed information.

\footnotetext{
3 A highly detailed discussion of the studies conducted so far on the neuronal grounds for the P300 potential and P3a and P3b subcomponents can be found in the quoted works of Polich (2007) and Polich and Criado (2006).
} 


\section{The P300 potential and polygraph examinations}

The first mentions about the usefulness of the P300 potential analysis for detection of concealed information can be found in the aforementioned article by Karis, Fabiani, Donchin (1984), which showed that important (e.g. earlier learnt) and rarely presented words evoke the P300 potential, while such a response is not observable in reaction to frequent words, unknown to the subject.

The first attempt at using the evoked potential analysis was made by Rosenfeld and his team (1987, after: Rosenfeld 2009). They observed a latent positive potential between $400 \mathrm{~ms}$ and $700 \mathrm{~ms}$ from the emergence of the stimulus in response to the presentation of an object in people who previously selected one out of nine objects, and who were asked to conceal their knowledge during the study. In the successive studies (Rosenfeld, Cantwell, Nasman, Wojdac, Ivanov, Mazzeri 1988 after: Rosenfeld 1999) a simulated crime procedure was used for the first time. During an experiment, the subjects received the task to take one of objects deposited in a box. People from the control group were assigned the task only to take a look at the hidden objects. The subsequent analyses showed that the average value of the P300 amplitude was significantly higher in the group of the 'guilty' subjects, who tried to conceal removing the object than in the group of the 'innocent' (Rosenfeld, Cantwell, Nasman, Wojdac, Ivanov, Mazzeri 1988, after: Rosenfeld 1999). At the same time, studies of the possibility of detecting deception through analysis of the P300 potential amplitude were conducted also by Farwell and Donchin, who presented their results at a conference of the Society for Psychophysiological Research already in 1986 (Farwell and Donchin 1986 after: Rosenfeld 2011), and published them five years later (Farwell, Donchin 1991).

It makes sense to note that for over two decades the studies devoted to the detection of concealed information based on the analysis of the P300 potential amplitude enjoyed plenty of interest from researchers, especially although not solely the ones gathered around Rosenfeld, the pioneer in the field. A number of studies proved the potential applicability in using a modified procedure, combining Guilty Knowledge Test and Control Questions Test as alternatives to screening tests (pre-employment tests aimed at examining the candidate's previous history, e.g. prior breaches of the law) (Rosenfeld, Angell, Johnson, Qian 1991; Johnson and Rosenfeld, 1992, after: Rosenfeld 2009). The procedure was also tested as a method for detecting simulated amnesia (Ellwanger, Rosenfeld, Sweet, Bhatt, 1996). Studies were conducted among others to de- 
tect: autobiographic data (among others: Rosenfeld, Rao, Soskins Miller 2003; Rosenfeld et al. 2008), knowledge related to face recognition (Meijer, Smulders, Merckelbach, Wolf 2007; Lefebvre, Marchand, Smith, Connolly 2009), information obtained during a simulated crime (among others: Rosenfeld, Soskins, Bosh, Ryan 2004; Winograd, Rosenfeld 2011), information obtained during a simulated crime committed in virtual reality (Mertens, Allen 2008; Hahm, Ji, Jeong, Oh, Kim, Sim, Lee 2009), and knowledge concerning terrorist attacks (Meixner, Rosenfeld 2011). Studies devoted to the discovery of acquired knowledge are inevitably a minority and only focus on elements that are central (e.g.: Winograd, Rosenfeld 2011) or repeatedly reiterated for better retention (e.g. Rosenfeld, Biroschak, Furedy 2006).

Another product of the studies conducted was the development of an array of procedures for disclosure of concealed information with the use of P300 potential amplitude analysis. The following subchapter is devoted to a detailed discussion of the proposed procedures.

\section{Development and modifications of EEG-supported study procedures used to disclose concealed information}

The first procedures for disclosing concealed information with the use of EEG were akin to the Guilty Knowledge Test used in polygraph examinations. Introduced as a necessary modification of the GKT in the first study to be conducted (Rosenfeld 1987) was the repeated presentation of each stimulus, that resulted from the need to collect numerous reactions in response to the same stimulus for later averaging. The successive studies (among others by Rosenfeld, Cantwell, Nasman, Wojdac, Ivanov, Mazzeri 1988, after: Rosenfeld 1999, Farwell and Donchin 1991) another modification was introduced, namely a stimulus category was added: besides the significant stimulus called 'probe' and irrelevant stimuli from the same category ('irrelevant'), this was the control stimulus - 'target' - unrelated to the crime, to which the subject was to react in a different manner (by pressing a different button) than in the case of all the remaining stimuli. This procedure known as 3SP (three stimulus protocol) has been used to this day (e.g. Mertens, Allen 2008; Hahm, Ji, Jeong, Oh, Kim, Sim, Lee 2009; Rosenfeld and Lui 2008).

A certain variety of this three-element test is the proposal to use a modified version of the Guilty Knowledge Test adjusted to screening tests (a combination of the Guilty Knowledge Test and Concealed Information Test) mentioned earlier. It assumes that the subject is presented with various behaviours, 
usually illegal or violating accepted norms - which is an element characteristic of screening tests (Rosenfeld, Angell, Johnson, Qian 1991; Johnson and Rosenfeld, 1992, after: Rosenfeld 2009) - rather than with elements of the committed crime (characteristic of GKT/CIT). However, characteristic of GKT/CIT, the structure of the test has been retained.

The latest modification proposed by Rosenfeld and his team (Rosenfeld et al. 2008) is the so-called Complex Trail Protocol (CTP). It is an answer to the main weakness of the three-stimulus procedure used earlier, namely vulnerability to counteraction. Rosenfeld and his collaborators managed to show that the use of simple methods described in greater detail further in the article significantly reduced efficiency of studies based on analysing the P300 potential (Rosenfeld et. al. 2004). The CTP was intended as an answer to such charges. During the study, a participant is presented with 4 types of stimuli: a significant stimulus ('probe', for example, the stolen object), non-significant stimuli ('irrelevants': other objects), a control stimulus (e.g. a sequence of five ones: 11111), and non-control stimuli - other sequences of five identical digits. The research conducted so far substantiates its high applicability and resilience to preventing detection (Rosenfeld et al. 2008; Sokolovsky, Rothenberg, Labkovsky, Meixner, Rosenfeld 2011). Moreover, it is claimed that additional analyses allow direct indication whether a given subject tried to resort to such methods (Rosenfeld, Labkovsky 2010; Meixner, Labkovsky, Rosenfeld, Winograd, Sokolovsky, Weishaar, Ullmann 2013).

\section{Guilty or innocent? Individual assessment procedures}

This subchapter discusses an array of methods that allow classification of subjects as 'guilty' or 'innocent'. This is important, as the procedure applied proves to influence the final result strongly.

The main drawback of the method of averaging potentials is that it results in obtaining a single evoked potential through the averaging of all reactions to the significant and irrelevant stimuli, which practically renders the application of any statistical tests impossible in reference to the results of a single person. In the first experiments (Rosenfeld 1987 and 1988, after: Rosenfeld 2011) classifying the subjects into two groups (guilty vs. innocent) the potentials were mostly assessed visually. The first attempts at application of statistical methods (e.g. Student's t-distribution tests) based on the distribution of individual evoked potentials proved to be highly inefficient due to a very high share of noise in the single iteration (Rosenfeld 2011). 
A solution to this problem, namely the use of the bootstrapping method, was proposed in the studies by Farwell and Donchin (1986, after: Wasserman and Bockenholt 1989; Farwell and Donchin 1991). Application of that method makes it possible to obtain an entire distribution of average potentials for the significant stimulus through sampling with replacement of sets that contain $\mathrm{X}^{4}$ individual iterations after the presentation of the significant stimulus and their subsequent averaging (Wasserman and Bockenholt 1989). The process may be repeated even 1000 times to acquire 1000 average potentials for the significant stimulus, and the whole procedure may be repeated for irrelevant and control stimuli as well (Wasserman and Bockenholt 1989). ${ }^{5}$ Having obtained the distribution of 1000 average passes for each stimulus category, various statistical tools can be applied to determine whether the reaction to the significant stimulus differs from the reaction to an irrelevant one. One of the first proposed methods for calculating the significance of this difference (Wasserman and Bockenholt 1989; Farwell and Donchin 1991) was the application of comparison of two correlation coefficients: the correlation coefficient between the significant and irrelevant stimulus, and the correlation coefficient between the significant and control stimuli. It was assumed that the ratio of inter-correlation between the significant and control stimulus will be higher in guilty individuals than the inter-correlation ratio between the significant and irrelevant stimulus. It was expected that the evoked potential following the significant stimulus in guilty individuals will be close to the classical P300 potential emerging after the control stimulus.

However, it was remarked (Rosenfeld et al. 1991, 2004; Rosenfeld 2011) that due to the different characteristics of the stimuli and the task related to them, it can be expected that the P300 potential following the control stimulus may differ from the P300 potential following the significant stimulus. The control stimulus is neutral for the subject, and its distinctive factor is only a different motoric reaction. The significant stimulus acquires a personal significance (ibidem) because the individual tries to conceal his or her knowledge related to that stimulus. This difference between two types of stimuli may result in the two potentials differing in the phase, and/or differ in the form or time of latency, as presented in the studies of Rosenfeld and his team (2004). Moreover, Rosenfeld (2011) emphasises that in the case of this method, the correla-

\footnotetext{
4 Wasserman and Bockenholt (1989) proposed the sampling of 10 single passes, currently the number of the passes drawn is equal to the number of accepted passes (containing no artefacts) for the significant stimulus.

5 Currently, the sampling is most often repeated 100 times, although as Rosenfeld remarked, 50 iterations are also sufficient (Rosenfeld 2011).
} 
tion coefficient between the potentials following the significant and irrelevant stimuli may be high in the group of guilty subjects, even if the amplitudes differ greatly one from the other, on the condition that the very shape of the potential is similar. ${ }^{6}$ Despite the method proposed by Farwell and Donchin to eliminate the last problem, Rosenfeld with Allen claim that the proposed remedy will not bring about the intended results in all cases (Rosenfeld 2011).

Another, and currently the most popular, method for individual diagnosis in detection of concealed information used in reference to the bootstrapped distribution of average potentials is the individual calculation of the difference between the value of P300 potential amplitude following the significant stimulus and the value of the P300 potential amplitude following the irrelevant stimulus (Rosenfeld et al. 1991, 2004, 2008, 2010; 2012; Sokolovsky et al. 2011). The result of these calculations is a distribution of, for example, 100 differences $^{7}$ between these two amplitudes. It is assumed that if, as result of these comparisons, the difference is greater than zero in $90 \%$ of cases, ${ }^{8}$ i.e. the amplitude of the P300 potential per significant stimulus is greater than the amplitude of the P300 potential per irrelevant stimulus, the subject is in the possession of knowledge concerning the given event, or, in other words, is guilty.

Besides the methods above described, literature also contains proposals of using other statistics to determine whether a person holds information that the investigator finds interesting. Rosenfeld and his team propose a certain modification of the method based on bootstrapping and the volume of amplitude, in which - instead of counting the number of cases where the amplitude following significant stimuli is greater than the amplitude following the irrelevant stimuli - tests based on the differences of the averages are used (Lui, Rosenfeld 2008). Another proposed method of assessing the evoked potentials is the use of methods based on Bayesian probability (Allen, Iacono, Danielson 1992). An advantage of this method is the possibility of employing a range of indicators to determine whether the reaction to the significant stimulus differs from the reaction to an irrelevant stimulus, for example, the simultaneous ap-

${ }^{6}$ Rosenfeld (2011) states that such a probability concerning the shape between the potential after the significant stimulus and the potential after an irrelevant stimulus can be observed among others in the studies by Farwell and Donchin (1991), Allen et al. (1992), and in the studies of Rosenfeld himself and with his team $(1991,2004)$.

7 The number depends on the number of the samplings made.

8 The threshold value is conventional: the value of $90 \%$ is most frequently used in the case of well learned material or autobiographical data, while in the case of data acquired during an event, it makes sense to lower the threshold to $80 \%$ (Rosenfeld: information acquired in person). 
plication of the volume of amplitude, the correlation coefficient, morphology of the potential, and/or reaction times in a behavioural task (ibidem). Studies conducted by Allen, Iacono (1997), and Mertens and Allen (2008) also demonstrate its higher efficiency (than e.g. the method proposed by Rosenfeld) in the detection of concealed information. Yet Rosenfeld (2011) remarks that neither of these studies is fully representative. In the first, the amplitude of the potential (part of the bootstrapping method based on the volume of the amplitude) was calculated according to the baseline-to-peak method instead of the peakto peak method more efficient in the case of disclosure of concealed information (see: Soskins, Rosenfeld, Niendam 2001; Meijer, Smulders, Merckelbach, Wolf 2007). In turn, all the methods used in the second study proved very low efficiency, and the high results in the one proposed by Allen concerned only certain cases of using detection prevention methods in the 3SP procedure.

\section{Procedures countering detection in the analysis of evoked potentials}

One of the grounds for seeking new methods of detecting concealed information was the vulnerability of polygraph examinations to methods upsetting detection applied during the examination. Examinations that made use of brain activity registration were to provide an answer to that weakness. For a long time, it was believed that due to the nature of registration of evoked potentials (changes of the order of a few hundred milliseconds) and the manner of stimulus presentation (very frequent and quick presentations), any detection frustrating methods is inconceivable (Ben-Shakhar, Elaad 2002; Lykken 1998, after: Rosenfeld 2011). Yet, as studies referring to the three-stimulus (3SP) method, highly popular until recently, show, the use of simple ruses that can be learnt independently decreases significantly the accuracy of conclusions drawn from the analyses of evoked potentials (Rosenfeld et al. 2004; Mertens, Allen 2008). The methods applied include:

1) increasing the intensity of the irrelevants (non-significant stimuli) for example through:

a. imagining being slapped in the face whenever the first irrelevant stimulus appears, straining the sphincter muscles whenever the second irrelevant stimulus appears, pressing the toes to the floor whenever the third irrelevant stimulus appears, and abstaining from any activity whenever the fourth irrelevant stimulus appears

2) increasing the significance of the control (target) stimulus e.g. through:

a. imagining being slapped in the face whenever the target stimulus appears

b. pressing the toes to the floor whenever the control stimulus appears. 
As has been mentioned, the results of this research was the development of a new procedure: the Complex Trial Protocol (CTP), which proved resilient to detection prevention methods used so far (Winograd, Rosenfeld 2011; Rosenfeld, Labkovsky 2010).

\section{The efficiency of disclosing concealed information with P300 potential analysis}

Literature sometimes features information about $100 \%$ efficiency of disclosing concealed information through the investigation of evoked potentials (Farwell 2012; Farwell, Richardson, Richardson 2013). Such assurance is related to the person of Lawrence Farwell, one of the pioneers of studies in detection of concealed information and the founder of the Brain Fingerprint Laboratory offering tests detecting concealed information, among others in criminal cases. The initiative was criticised by other researchers (e.g. Rosenfeld 2005) as too quick an attempt at monetising research results. Moreover, researchers were critical about Farewell's assurances about the excellent efficiency of his method and the studies he conducted, and pointed to a range of errors in his interpretation of results, and challenged the method of result calculation (Meijer, Ben-Shakhar, Verschuere, Donchin 2013). For that reason, the results of Farewell's research are not accounted for in this chapter. Table 3 presents a review of EEG-enabled studies on disclosure of concealed information conducted so far.

Despite a plethora of experiments conducted so far, it is very difficult to determine the general efficiency of the method of detecting deception with the use of evoked potentials. An aspect that renders the above more difficult is the fact that the studies made use of various experimental procedures (both classical 3SPs, and the later-CTPs), different methods of assigning subjects to the groups of 'the innocent' and 'the guilty', and the fact that some of the studies were geared towards researching the methods of frustrating such detection. Analysis of the data available demonstrates that the studies making use of the P300 potential recording do not attain a significantly higher efficiency than polygraph examinations, although the results can be considered promising. It was worth adding that there is a shortage of field studies on the efficiency of detecting concealed information with the use of the P300 potential analysis. One of the few studies, conducted still early in the 1990s, points to its efficiency being lower than that of polygraph examinations (Miyake, Mizutanti, Yamahura 1993). 
Table 3. Efficiency of selected concealed information detection studies making use of the P300 potential analysis

\begin{tabular}{|c|c|c|c|c|c|}
\hline \multirow{2}{*}{$\begin{array}{c}\text { data } \\
\text { source }\end{array}$} & \multirow{2}{*}{$\begin{array}{l}\text { comments on the subject of the } \\
\text { study, its procedure, and meth- } \\
\text { ods of data analysis }\end{array}$} & \multicolumn{2}{|c|}{ 'innocent' } & \multicolumn{2}{|c|}{ 'guilty' } \\
\hline & & correct & incorrect & correct & incorrect \\
\hline \multirow{4}{*}{$\begin{array}{l}\text { Mertens, } \\
\text { Allen } \\
2008^{*}\end{array}$} & $\begin{array}{c}\text { virtual simulated crime - well } \\
\text { learned material - bootstrapping - } \\
\text { correlations }^{\text {semses }}\end{array}$ & $27 \%$ & $13 \%$ & $44 \%$ & $0 \%$ \\
\hline & $\begin{array}{c}\text { virtual simulated crime - well } \\
\text { learned material - Bayesian prob- } \\
\text { ability }\end{array}$ & $94 \%$ & $6 \%$ & $47 \%$ & $53 \%$ \\
\hline & $\begin{array}{c}\text { virtual simulated crime - well } \\
\text { learned material - bootstrapping - } \\
\text { peak-to-peak }\end{array}$ & $100 \%$ & $0 \%$ & $47 \%$ & $53 \%$ \\
\hline & $\begin{array}{l}\text { virtual simulated crime - well } \\
\text { learned material - after applica- } \\
\text { tion of methods frustrating detec- } \\
\text { tion }\end{array}$ & $\begin{array}{l}\text { from } 27 \\
\text { to } 100 \%\end{array}$ & $\begin{array}{l}\text { from } 0 \text { to } \\
13 \%\end{array}$ & $\begin{array}{l}\text { from } 7 \\
\text { to } 27 \%\end{array}$ & $\begin{array}{l}\text { from } 0 \text { to } \\
\qquad 89 \%\end{array}$ \\
\hline $\begin{array}{l}\text { Hu, } \\
\text { Rosenfeld } \\
2012^{* *}\end{array}$ & $\begin{array}{l}\text { simulated crime - the studies were } \\
\text { focused on ensuring } 100 \% \text { efficien- } \\
\text { cy in innocent individuals }\end{array}$ & $100 \%$ & $0 \%$ & $58 \%$ & $42 \%$ \\
\hline $\begin{array}{l}\text { Soskins, } \\
\text { Rosenfeld, } \\
\text { Niendam } \\
2001\end{array}$ & $\begin{array}{l}\text { autobiographic information - the } \\
\text { studies were focused on compar- } \\
\text { ing various ways of preparing sig- } \\
\text { nal for analyses - the results pre- } \\
\text { sented concern the best method }\end{array}$ & $100 \%$ & $0 \%$ & $100 \%$ & $0 \%$ \\
\hline $\begin{array}{l}\text { Rosenfeld, } \\
\text { Soskins, } \\
\text { Bosh, } \\
\text { Ryan 2004 } \\
\end{array}$ & simulated crime & $91 \%$ & $9 \%$ & $92 \%$ & $8 \%$ \\
\hline Rosenfeld & $\begin{array}{l}\text { Autobiographic information - new } \\
\text { procedure: Complex Trial Protocol }\end{array}$ & $92 \%$ & $8 \%$ & $100 \%$ & $0 \%$ \\
\hline $\begin{array}{l}\text { Labkovsky } \\
2010\end{array}$ & $\begin{array}{l}\text { Autobiographic information - new } \\
\text { procedure: Complex Trial Proto- } \\
\text { col accounting for counteraction } \\
\text { methods }\end{array}$ & $93 \%$ & $8 \%$ & $100 \%$ & $0 \%$ \\
\hline
\end{tabular}




\begin{tabular}{|c|c|c|c|c|c|}
\hline $\begin{array}{l}\text { Mexiner, } \\
\text { Rosenfeld } \\
2011\end{array}$ & $\begin{array}{l}\text { Information concerning a terrorist } \\
\text { attack - knowledge obtained ran- } \\
\text { domly, yet with enforced multiple } \\
\text { repetition by the subject (new pro- } \\
\text { cedure: Complex Trial Protocol) }\end{array}$ & $100 \%$ & $0 \%$ & $83 \%$ & $17 \%$ \\
\hline \multirow{2}{*}{$\begin{array}{l}\text { Winograd, } \\
\text { Rosenfeld } \\
2011\end{array}$} & $\begin{array}{l}\text { simulated crime - no active re- } \\
\text { membering (only the object of the } \\
\text { theft) (new procedure: Complex } \\
\text { Trial Protocol) }\end{array}$ & $92 \%$ & $8 \%$ & $83 \%$ & $17 \%$ \\
\hline & $\begin{array}{l}\text { simulated crime - no active re- } \\
\text { membering (only the object of the } \\
\text { theft) , accounting for counterac- } \\
\text { tion methods (new procedure: } \\
\text { Complex Trial Protocol) }\end{array}$ & $92 \%$ & $8 \%$ & $100 \%$ & $0 \%$ \\
\hline $\begin{array}{l}\text { Farwell, } \\
\text { Donchin } \\
1991^{* m s}\end{array}$ & $\begin{array}{c}\text { simulated crime - well learned } \\
\text { material }\end{array}$ & $85 \%$ & $0 \%$ & $90 \%$ & $0 \%$ \\
\hline
\end{tabular}

"In the presentation, the result of Martens's studies were broken down into the procedures of classifying people into 'guilty' and 'innocent' applied.

"Presented are the averaged results from two experimental groups.

The results obtained with this method of classification did not total up to $100 \%$, as this method accounts for the 'unresolved' category.

The results obtained with this method of classification did not total up to $100 \%$, as this method accounts for the 'unresolved' category.

Despite testing the efficiency of detecting concealed information through the analysis of the amplitude of the P300 potential, investigations have also been conducted to test the efficiency of drawing conclusions based on the combined measures falling back on the registration of the evoked potentials and the functioning of the autonomous nervous system (Ambach, Bursch, Stark, Vaitl 2010). Although the use of these methods obtained only a modest increase in the efficiency of drawing conclusions, it should be remarked that conducting examinations in these two methods, one after the other, may assure a higher efficiency of disclosing concealed information (Meijer, Selle, Elber, Ben-Shakhar 2014). 


\section{Theoretical grounds for the development of the P300 potential in concealed information tests}

As attempted to describe in the subchapter on the study of evoked potentials, the P300 potential is a complex electrical response of the brain, and depends on numerous factors. For that reason, the determination of clear grounds for the occurrence of the P300 potential in the concealed information test causes certain difficulties.

On the one hand, many researchers emphasise that the studies in detecting concealed information are based on the orientative reaction (Vrij 2008; Rosenfeld, Hu Pederson 2012). Yet on the other, falling back on the views of Donchin (1981), one can associate the P300 amplitude in the studies in detection of concealed information with memory processes, including recognition (the $\mathrm{P} 3 \mathrm{~b}$ subcomponent) (Lui, Rosenfeld 2008). This is suggested by the selection of the Pz electrode by majority of researchers dealing with the detection of concealed information, as this is where the amplitude is greatest (e.g.: Rosenfeld et al. 1991). For that reason, it was believed that the use of components of the P300based detection of concealed information would allow achievement of better results, while analysing the occipital-parietal component than in the case of the frontal-central one (Lui, Rosenfeld 2008). However, the studies conducted suggested an inverse relationship, as they prove that a far superior robustness of conclusions was achieved from the occipital-parietal component (ibidem). Connecting the activity in the area with the P3a subcomponent, ${ }^{9}$ researchers emphasise the key significance of the processes of attention and inhibition of sincere (i.e. non-deceptive) answer.

Supporting the key significance of inhibition in the process of disclosing information are also the data that prove that, much like in the case of the polygraph (see: Gustfson, Ore 1963), realisation of the need to concealed information, i.e. an increase in motivation, triggers an increase in the efficiency of disclosing concealed information by analysing the P300 potential (Rosenfeld et al. 2012). ${ }^{10}$

\footnotetext{
9 One can only remark that despite the frontal-central localisation of the component, attention is drawn to its very long latency (in the study quoted above, the latency ranged from $220 \mathrm{~ms}$ to $664 \mathrm{~ms}$ for a procedure composed of two significant stimuli), while many researchers emphasise a very short time of latency of the P3a subcomponent.

${ }^{10}$ It is worth, however, to note that the data are not unambiguous, as earlier studies (Verschuere, Rosenfeld, Winograd, Labkovsky, Wiersema 2009) did not point to a link between an increase in the awareness of an act of deceit and the amplitude, although they pointed to a positive correlation with the efficiency of drawing conclusions.
} 


\section{Conclusion}

It is worth noting that the studies that make use of evoked potentials analysis for the detection of concealed information have continued to be no more than an experimental method that still requires plenty of attention on behalf of researchers. Attempts at commercialisation of results of the investigations and their practical use by Farewell encountered major criticism from the academic circles. The weakness of the presented studies, from the point of view of the potential use of the method in actual criminal cases as an alternative to the polygraph, is the focus on the disclosure of autobiographic data (Rosenfeld et al. 2003, 2008) and data learnt very well during special sessions aimed at the retention of the memorised material (see: Mertens, Allen 2008). Such grounds make drawing of conclusions about the potential use of the method, for example in investigations, difficult. On the other hand, the higher resilience to counteraction (when the data related to the new CTP protocol are taken into account), and falling back on the functioning of the peripheral nervous system, and the ever better investigated reasons for the development of the P300 potential encouraging to conduct further investigations of this method of detecting concealed information. What seems to be an advantage of polygraph examinations from the practical point of view is their rich history that encompasses both experimental and laboratory research, as well as their extensive scope of application. In turn, studies that make use of the EEG seem to have better theoretical foundations, as they record bioelectrical processes related to the cognitive processes of processing information.

\section{References}

Allen J.J.B., Iacono W.G. (1997), A comparison of methods for the analysis of event-related potentials in deception-detection, Psychophysiology, 34, pp. 234-240.

Allen J.J., Iacono W.G., Danielson K.D. (1992), The identification of concealed memories using the event-related potential and implicit behavioral measures: A methodology for prediction in the face of individual differences, Psychophysiology, 29, pp. 504-522.

Ambach W., Bursch S., Stark R., Vaitl D. (2010), A Concealed Information Test with multimodal measurement, International Journal of Psychophysiology, 75 (3), pp. 258-267. 
Ben-Shakhar G., Elaad E. (2002), The Guilty Knowledge Test (GKT) as an application of psychophysiology: future prospects and obstacles. P300 in detecting concealed information, [in:] M. Kleiner (ed.), Handbook of Polygraph Testing (pp. 87-102), San Diego, Academic Press.

Dawson G.D. (1947), Cerebral responses to electrical stimulation of peripheral nerve in man, Journal of Neurology, Neurosurgery \& Psychiatry,10(3), pp. 134140.

Donchin E. (1981), Surprise!....Surprise?, Psychophysiology, 18, pp. 493-513.

Duncan-Johnson C.C., Donchin E. (1977), On quantifying surprise: the variation of event-related potentials with subjective probability, Psychophysiology, 14(5), pp. 456-467.

Ellwanger J., Rosenfeld J.P., Sweet J.J., Bhatt M. (1996), Detecting simulated amnesia for autobiographical and recently learned information using the P300 event-related potential, International Journal of Psychophysiology, 23(1-2), pp. 9-23.

Fabiani M., Gratton G., Coles M.G.H. (2000), Event related brain potentials: Methods, theory and applications, [in:] J. Cacioppo, L. Tassinary, G. Berntson (eds), Handbook of Psychophysiology (pp. 53-84), New York, Cambridge University Press.

Farwell L.A. (2012), Brain fingerprinting: a comprehensive tutorial review of detection of concealed information with event-related brain potentials, Cognitive Neurodynamics, 6, pp. 115-154.

Farwell L.A., Donchin E. (1986), The brain detector: P300 in the detection of deception, Psychophysiology, 24, p. 34 (abstract).

Farwell L.A., Donchin E. (1991), The truth will out: interrogative polygraphy ("lie detection') with event-related brain potentials, Psychophysiology, 28(5), pp. 531-547.

Farwell L.A., Richardson D.C., Richardson G.M. (2013), Brain fingerprinting field studies comparing P300-MERMER and P300 brainwave responses in the detection of concealed information, Cognitive neurodynamics, 7(4), pp. 263-299.

Gustafson L.A., Orne M.T. (1963), Effects of heightened motivation on the detection of deception, Journal of Applied Psychology, 47(6), pp. 408-411.

Hahm J., Ji H.K., Jeong J.Y., Oh D.H., Kim S.H., Sim K.B., Lee J.H. (2009), Detection of concealed information: combining a virtual mock crime with 
a P300-based Guilty Knowledge Test, Cyberpsychology \& behavior: the impact of the Internet, multimedia and virtual reality on behavior and society, 12(3), pp. 269-275.

Hu X., Rosenfeld J.P. (2012), Combining the P300-complex trial-based Concealed Information Test and the reaction time-based autobiographical Implicit Association Test in concealed memory detection, Psychophysiology, 49(8), pp. 1090-1100.

Jaśkowski P. (2009), Neuronauka poznawcza. Jakmózg tworzy umyst, Warszawa, Vizja Press \& IT.

Johnson R. (1993), On the neural generators of the P300 component of the event-related potential, Psychophysiology, 30(1), pp. 90-97.

Johnson M.M., Rosenfeld J.P. (1992), Oddball-evoked P300-based method of deception detection in the laboratory II: Utilization of non-selective activation of relevant knowledge, International Journal of Psychophysiology, 12(3), pp. 289-306.

Karis D., Fabiani M., Donchin E. (1984), 'P300' and memory: Individual differences in the von Restorff effect, Cognitive Psychology, 16(2), pp. 177-216.

Lefebvre C.D., Marchand Y., Smith S.M., Connolly J.F. (2009), Use of eventrelated brain potentials (ERPs) to assess eyewitness accuracy and deception, International Journal of Psychophysiology, 73(3), pp. 218-225.

Lui M., Rosenfeld J.P. (2008), Detection of deception about multiple, concealed, mock crime items, based on a spatial-temporal analysis of ERP amplitude and scalp distribution, Psychophysiology, 45(5), pp. 721-730.

Lykken D.T. (1998), A Tremor in the blood: Uses and abuses of the lie detector, 2nd ed, New York, Plenum Press.

Meijer E.H., Ben-Shakhar G., Verschuere B., Donchin E. (2013), A comment on Farwell (2012): brain fingerprinting: a comprehensive tutorial review of detection of concealed information with event-related brain potentials, Cognitive neurodynamics, 7(2), pp. 155-158.

Meijer E.H., Selle N.K., Elber L., Ben-Shakhar G. (2014), Memory detection with the Concealed Information Test: A meta-analysis of skin conductance, respiration, heart rate, and P300 data, Psychophysiology, 51(9), pp. 879-904.

Meijer E.H., Smulders F.T.Y., Merckelbach H.L.G.J., Wolf A.G. (2007), The P300 is sensitive to concealed face recognition, International Journal of Psychophysiology, 66(3), pp. 231-237. 
Meixner J.B., Labkovsky E., Rosenfeld J.P., Winograd M., Sokolovsky A., Weishaar J., Ullmann T. (2013), P900: a putative novel ERP component that indexes countermeasure use in the P300-based concealed information test, Applied Psychophysiology and Biofeedback, 38(2), pp. 121-132.

Meixner J., Rosenfeld J. (2011), A mock terrorism application of the P300based concealed information test, Psychophysiology, 48, pp. 149-154.

Mertens R., Allen J.J.B. (2008), The role of psychophysiology in forensic assessments: deception detection, ERPs, and virtual reality mock crime scenarios, Psychophysiology, 45(2), pp. 286-298.

Miyake Y., Mizutanti M., Yamahura T. (1993), Event related potentials as an indicator of detecting information in field polygraph examinations, Polygraph, 22(2), pp. 131-149.

Obermann C.E. (1939), The effect on the Berger rhythm of mild affective states, The Journal of Abnormal and Social Psychology, 34(1), 84.

Polich J. (2003), Theoretical overview of P3a and P3b (pp. 83-98), Springer US.

Polich J. (2007), Updating P300: An integrative theory of P3a and P3b, Clinical Neurophysiology, 118, pp. 2128-2148.

Polich J., Criado J.R. (2006), Neuropsychology and neuropharmacology of P3a and P3b, International Journal of Psychophysiology, 60(2), pp. 172-185.

Rosenfeld J.P. (1999), Event-related potential in detection of deception, www. psych.northwestern.edu/ rosenfel/publications.html.

Rosenfeld J.P. (2005), 'BRAIN FINGERPRINTING': A Critical Analysis, The Scientific Review of Mental Health Practice, 4(1), pp. 20-37.

Rosenfeld J.P. (2011), P300 in Detecting Concealed Information, [in:] B. Verschuere, G. Ben Shakhar, E. Meijer (eds), Memory detection: Theory and application of the concealed information test, Cambridge, Cambridge University Press.

Rosenfeld J.P., Angell A., Johnson M., Qian J.H. (1991), An ERP-based, control-question lie detector analog: algorithms for discriminating effects within individuals' average waveforms, Psychophysiology, 28(3), pp. 319-335.

Rosenfeld J.P., Biroschak J.R., Furedy J.J. (2006), P300-based detection of concealed autobiographical versus incidentally acquired information in target 
and non-target paradigms, International Journal of Psychophysiology, 60(3), pp. 251-259.

Rosenfeld J.P., Cantwell B., Nasman V.T., Wojdac V., Ivanov S., Mazzeri L. (1988), A modified, event-related potential-based guilty knowledge test, International Journal of Neuroscience, 1988 (24), pp. 157-161.

Rosenfeld J.P., Hu X., Pederson K. (2012), Deception awareness improves P300-based deception detection in concealed information tests, International Journal of Psychophysiology, 86(1), pp. 114-121

Rosenfeld J.P., Labkovsky E. (2010), New P300-based protocol to detect concealed information: Resistance to mental countermeasures against only half the irrelevant stimuli and a possible ERP indicator of countermeas, Psychophysiology, 47, pp. 1002-1010.

Rosenfeld J.P., Labkovsky E., Winograd M., Lui M.A., Vandenboom C., Chedid E. (2008), The Complex Trial Protocol (CTP), A new, countermeasure-resistant, accurate, P300-based method for detection of concealed information, Psychophysiology, 45(6), pp. 906-919.

Rosenfeld J.P., Meixner J., Kievit R.A., Schendan H.E., Ganis G. (2011), Lying in the scanner: Covert countermeasures disrupt deception detection by functional magnetic resonance imaging, NeuroImage, 55, pp. 312-319.

Rosenfeld J.P., Nasman V.T., Whalen I., Cantwell B., Mazzeri L. (1987), Late vertex positivity in event-related potentials as a guilty knowledge indicator: a new method of lie detection, International Journal of Neuroscience, 34, pp. 125-129.

Rosenfeld J.P., Rao A., Soskins M., Miller A.R. (2003), Scaled P300 scalp distribution correlates of verbal deception in an autobiographical oddball paradigm: Control for task demand, Journal of Psychophysiology, 17(1), pp. 14-22.

Rosenfeld J.P., Soskins M., Bosh G., Ryan A. (2004), Simple, effective countermeasures to P300-based tests of detection of concealed information, Psychophysiology, 41(2), pp. 205-219.

Schmitt R. (1993), Aktywność elektryczna mózgu, [in:] T. Sosnowski, K. Zimmer (eds), Metody psychofizjologiczne w badaniach psychologicznych: praca zbiorowa (P. Zaborowski, A. Krzymowska (translators), pp. 101-136), Warszawa, Wydawnictwo Naukowe PWN.

Sokolovsky A., Rothenberg J., Labkovsky E., Meixner J., Rosenfeld J.P. (2011), A novel countermeasure against the reaction time index of countermeasure 
use in the P300-based complex trial protocol for detection of concealed information, International Journal of Psychophysiology, 81(1), pp. 60-63.

Soskins M., Rosenfeld J.P., Niendam T. (2001), Peak-to-peak measurement of P300 recorded at $0.3 \mathrm{~Hz}$ high pass filter settings in intraindividual diagnosis: Complex vs. simple paradigms, International Journal of Psychophysiology, 40(2), pp. 173-180.

Sosnowski T. (2000), Psychofizjologia, [in:] J. Strelau (ed.), Psychologia. Podręcznik akademicki (vol. 1, pp. 131-178), Gdańsk, Gdańskie Wydawnictwo Psychologiczne.

Squires K.C., Squires N.K., Hillyard S.A. (1975), Decision-related cortical potentials during an auditory signal detection task with cued observation intervals, Journal of experimental psychology. Human perception and performance, 1(3), pp. 268-279.

Sutton S., Braren M., Zubin J., John E.R. (1965), Evoked-potential correlates of stimulus uncertainty, Science, 150 (700), pp. 1187-1188.

Szelenberger W. (2001), Potencjaty wywołane, Warszawa, Wydawnictwo Elmiko.

Vrij A. (2008), Detecting Lies and Deceit. Pitfalls and Opportunities (2nd ed), Chichester: John Wiley \& Sons Ltd.

Wasserman S., Bockenholt U. (1989), Bootstrapping: applications to psychophysiology, Psychophysiology, 26(2), pp. 208-221.

Winograd M., Rosenfeld J.P. (2011), Mock crime application of the Complex Trial Protocol (CTP) P300-based Concealed Information Test, Psychophysiology, 48, pp. 155-161. 